\title{
DSAM lifetime measurements for the chiral pair in ${ }^{194} \mathrm{TI}$
}

\author{
P.L. Masiteng ${ }^{1,2,3, a}$, A.A. Pasternak ${ }^{4}$, E.A. Lawrie ${ }^{1, b}$, O. Shirinda ${ }^{1}$, J.J. Lawrie ${ }^{1}$, R.A. Bark ${ }^{1}$, S.P. Bvumbi ${ }^{1,2,3}$, \\ N.Y. Kheswa ${ }^{1}$, R. Lindsay ${ }^{2}$, E.O. Lieder ${ }^{1}$, R.M. Lieder ${ }^{1}$, T.E. Madiba ${ }^{1,2}$, S.M. Mullins ${ }^{1}$, S.H.T. Murray ${ }^{1}$, \\ J. Ndayishimye ${ }^{1,5}$, S.S. Ntshangase ${ }^{1,6}$, P. Papka ${ }^{1,5}$, and J.F. Sharpey-Schafer ${ }^{1,2}$ \\ 1 iThemba LABS, National Research Foundation, PO Box 722, 7129 Somerset West, South Africa \\ 2 University of the Western Cape, Private Bag X17, 7535 Bellville, South Africa \\ 3 University of Johannesburg, PO Box 524, Auckland Park, South Africa \\ 4 A.F. Ioffe Physical-Technical Institute, 194021 St.-Petersburg, Russia \\ 5 Department of Physics, University of Stellenbosch, Private Bag X1, 7602 Matieland, South Africa \\ 6 Department of Physics, University of Cape Town, Private Bag, 7701 Rondebosch, South Africa
}

Received: 21 September 2015 / Revised: 15 December 2015

Published online: 12 February 2016

(c) The Author(s) 2016. This article is published with open access at Springerlink.com Communicated by A. Jokinen

\begin{abstract}
Most important for the identification of chiral symmetry in atomic nuclei is to establish a pair of bands that are near-degenerate in energy, but also in $B(M 1)$ and $B(E 2)$ transition probabilities. Dedicated lifetime measurements were performed for four bands of ${ }^{194} \mathrm{Tl}$, including the pair of four-quasiparticle chiral bands with close near-degeneracy, considered as a prime candidate for best chiral symmetry pair. The lifetime measurements confirm the excellent near-degeneracy in this pair and indicate that a third band may be involved in the chiral symmetry scenario.
\end{abstract}

Chiral systems can exist in nuclei with triaxially deformed shape. Such nuclei rotate collectively predominantly around their intermediate axis. Should the valence nucleons have both particle and hole nature, their singleparticle angular momenta would align along the short and long nuclear axes, respectively. Then the total angular momentum of the nucleus will have large projections along the three major nuclear axes, forming a left-handed or a right-handed systems and exhibiting chiral symmetry.

Nuclear chiral symmetry generates a pair of rotational bands with the same parity and with near-degenerate properties; for instance they have similar excitation energies, alignments, and reduced $B(M 1)$ and $B(E 2)$ transition probabilities [1]. Most of the chiral bands known to date have been identified based on similarity in the excitation energy and alignments, but very often the most crucial chirality test (see ref. [2]), i.e. the similarity in the $B(M 1)$ and $B(E 2)$ transition rates remain outstanding, because it needs dedicated lifetime measurements.

The formation of more than one chiral system in the same nucleus is a very rare event. To date chiral multiplets were proposed in only two nuclei, ${ }^{133} \mathrm{Ce}[3]$ and ${ }^{103} \mathrm{Rh}[4]$,

\footnotetext{
a e-mail: plmasiteng@uj.ac.za

b e-mail: elena@tlabs.ac.za
}

although both cases are still to be confirmed by lifetime measurements.

In this work lifetime measurements were performed for four bands in ${ }^{194} \mathrm{Tl}$ [5], including the only fourquasiparticle chiral pair known to date. This is also the only chiral pair for which chirality persists through a backbend. Furthermore, this chiral pair shows perhaps the best near-degeneracy found to date [6].

The ${ }^{181} \mathrm{Ta}\left({ }^{18} \mathrm{O}, 5 \mathrm{n}\right)$ reaction was employed at a beam energy of $91 \mathrm{MeV}$. The target was a $1 \mathrm{mg} / \mathrm{cm}^{2}{ }^{181} \mathrm{Ta}$ foil with a thick Bi layer evaporated on the back. The recoils were completely stopped in the Bi backing. The emitted $\gamma$-rays were detected with the AFRODITE array $[7,8]$ at iThemba LABS, comprising 9 Compton-suppressed clover detectors, and 6 LEPS detectors. The trigger required 3 coincident $\gamma$-rays, with at least two detected in the clovers. Four clover detectors were arranged at $45^{\circ}$, another four were placed at $135^{\circ}$, while the remaining detectors were situated at $90^{\circ}$ with respect to the beam direction.

Data were sorted into two asymmetric matrices, with the $\gamma$-ray energies detected at $45^{\circ}$ and at $135^{\circ}$, respectively, stored into one axis, while the coincident $\gamma$-ray energies registered at any angle were stored into the second axis. Gated, background-subtracted, forward $\left(45^{\circ}\right)$ and backward $\left(135^{\circ}\right)$ spectra were used to analyze the Doppler 

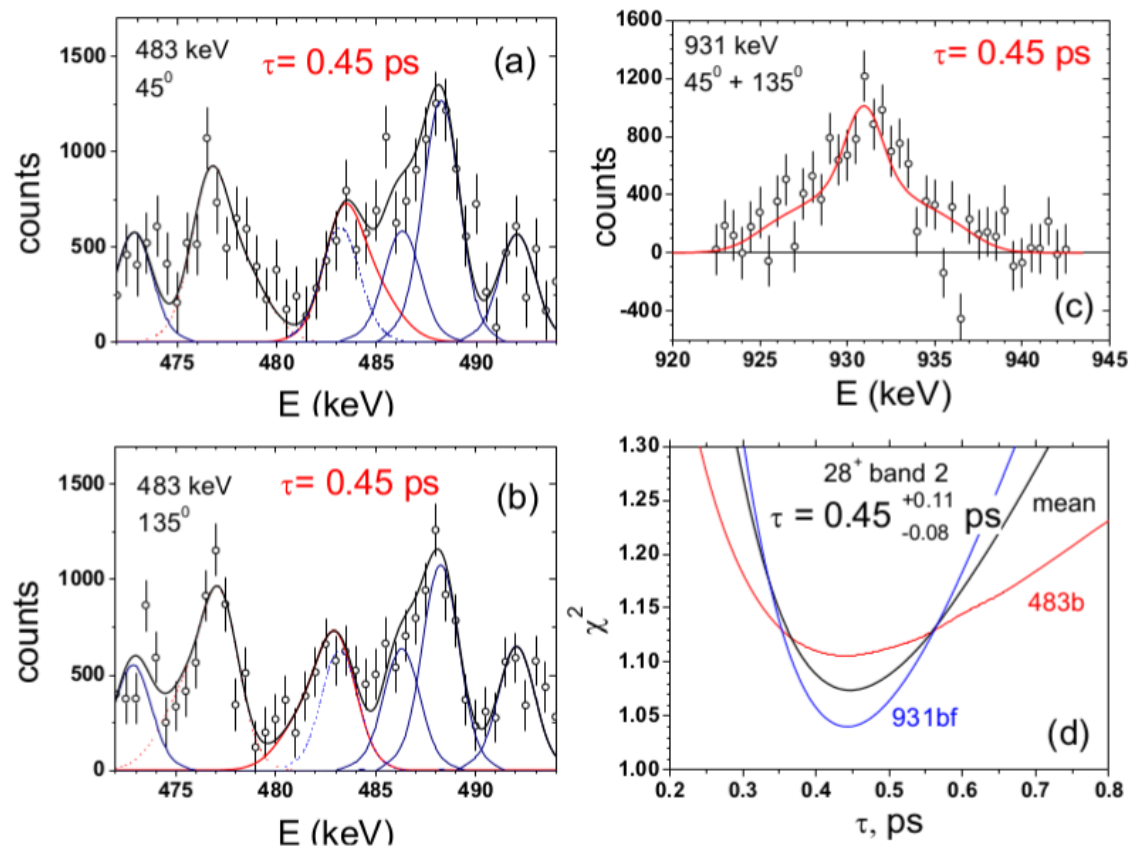

Fig. 1. (Color online) Line shape analysis of the transitions de-exciting the $28^{+}$level of Band 2 . Analysis of the $483 \mathrm{keV}$ peak at (a) forward and (b) backward, angles, and (c) analysis of the $931 \mathrm{keV}$ peak in the spectrum that is a sum of the forward and backward spectra. The fit of the Doppler broadened peak of interest is shown with red solid line. Peaks without Doppler broadening are fitted with apparatus line shapes, shown in blue. The position of the stopped component of the $483 \mathrm{keV}$ peak is plotted by the dashed blue line. The black solid line shows the fit for all peaks. In panel (d) the $\chi^{2}$ functions from the analysis of the spectra shown in (b) and (c) are plotted.

broadening of the peaks using the Doppler Shift Attenuation Method (DSAM). The analysis involved gating on intense transitions lying below the transition of interest, which is the "wide gate on transition below" method [10], and implies that the side feeding patterns have to be simulated.

The DSAM analysis was performed using the programs COMPA, GAMMA, and SHAPE [9], described in detail in [10-13], and previously applied with AFRODITE data $[14,15]$. Monte-Carlo methods were used to simulate the entry state and the side feeding cascades to the level of interest. Statistical $E 1, M 1$ and $E 2$ transitions, $E 2$ bands including superdeformed bands, and stretched $M 1$ bands generated by particle-hole excitations were considered. Parameters for the side feeding were taken from the literature or extracted from the present data, using the procedure described in $[10,16]$.

Lifetimes were determined step by step, starting from the highest-energy level of a band. Examples of DSAM analysis are shown in figs. 1 and 2. The line shapes for the $\gamma$-rays de-exciting the higher-spin levels show larger Doppler broadening. The $28^{+}$level de-excites by the 483 and $931 \mathrm{keV}$ transitions. The difference in the Doppler broadening at forward and backward angles is clearly distinguishable even for the medium-energy $483 \mathrm{keV}$ peak - note the different slopes of the low energy part of this peak, shown with red solid line in panels (a) and (b) of fig. 1. Much larger Doppler broadening is observed for the $931 \mathrm{keV}$ peak, which is symmetric in the sum of the for- ward and backward spectra, shown in panel (c) of fig. 1 . Panel (d) illustrates the corresponding $\chi^{2}$ functions for the fits of the spectra in panels (b) and (c), and the extracted lifetime of $\tau=0.45_{-0.08}^{+0.11} \mathrm{ps}$. The asymmetric error bars are determined at $\chi_{\min }^{2}+1 / K$, where $K$ denotes the degrees of freedom of the fit.

DSAM measurements can also yield results for peaks with small Doppler broadening. Such an analysis is described in detail in [17]. To underline the sensitivity of the method, as a second example, DSAM analysis for a peak with a small Doppler broadening is shown in fig. 2. The $22^{-}$level of Band 1 is fed by a direct cascade, as well as by side feeding. The line shape of the $563 \mathrm{keV}$ transition, de-exciting this level, shows a small Doppler broadening, see fig. 2(a), due to the small fraction of the sidefeeding with respect to the direct feeding. Nevertheless the spectrum has very good statistics, which allows a distinct minimum of the $\chi^{2}$ function to be obtained, see panel (b). The extracted lifetime result can be further tested. In panel (c) a spectrum is made as a difference between the backward and forward spectra. This spectrum is very sensitive to Doppler broadening. Should the peak at the forward (backward) angle show broadening at the high (low)-energy side, the difference spectrum would produce a characteristic shape, which crosses zero at the centroid of the peak. This curve is then analyzed to extract the lifetime using the flight-fraction method (for more details on this method see [18]) illustrated in fig. 2(d). The two 

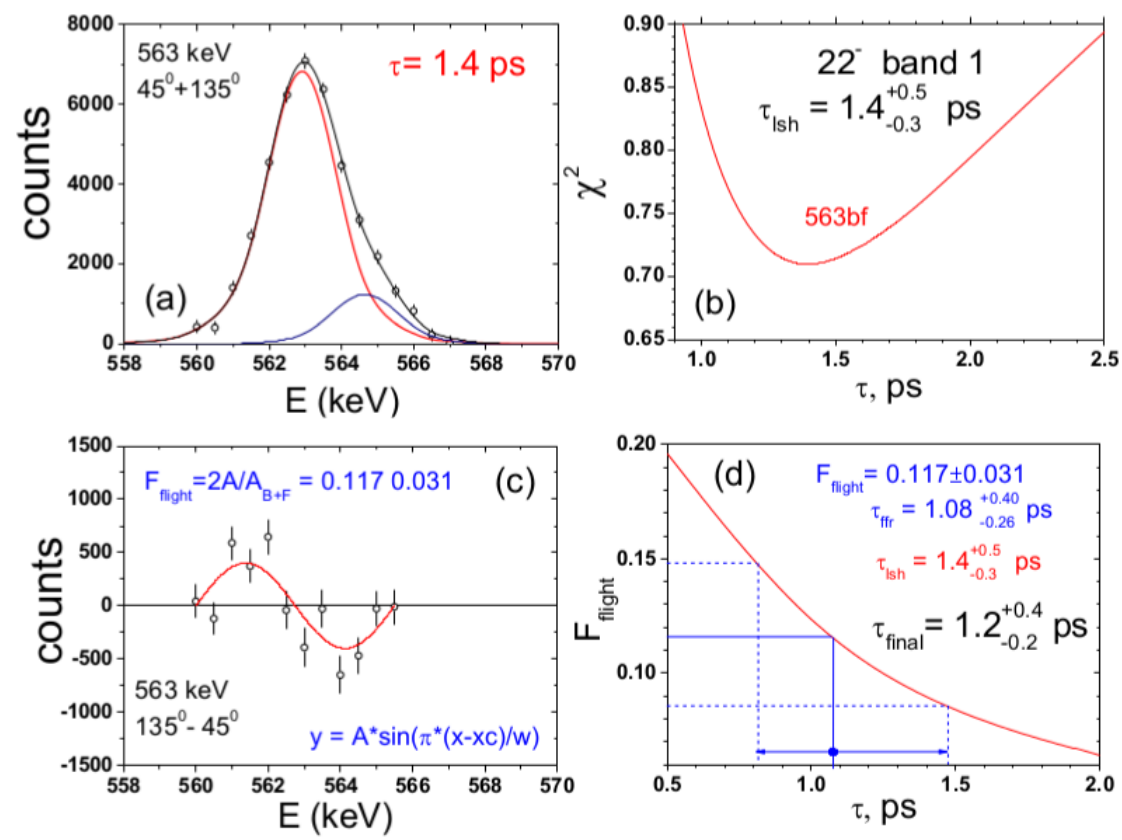

Fig. 2. (Color online) Line shape analysis of the $563 \mathrm{keV}$ transition de-exciting the $22^{-}$level of Band 1 . In panel (a) a spectrum that is a sum of the forward and backward spectra is shown, the notation is as in fig. 1 . The $\chi^{2}$ analysis for this peak is plotted in panel (b). Panels (c) and (d) illustrate the flight-fraction analysis, with an analysis of the spectrum that is a difference of the backward and forward spectra (c), and the extraction of the lifetime (d).

\section{Band 2}

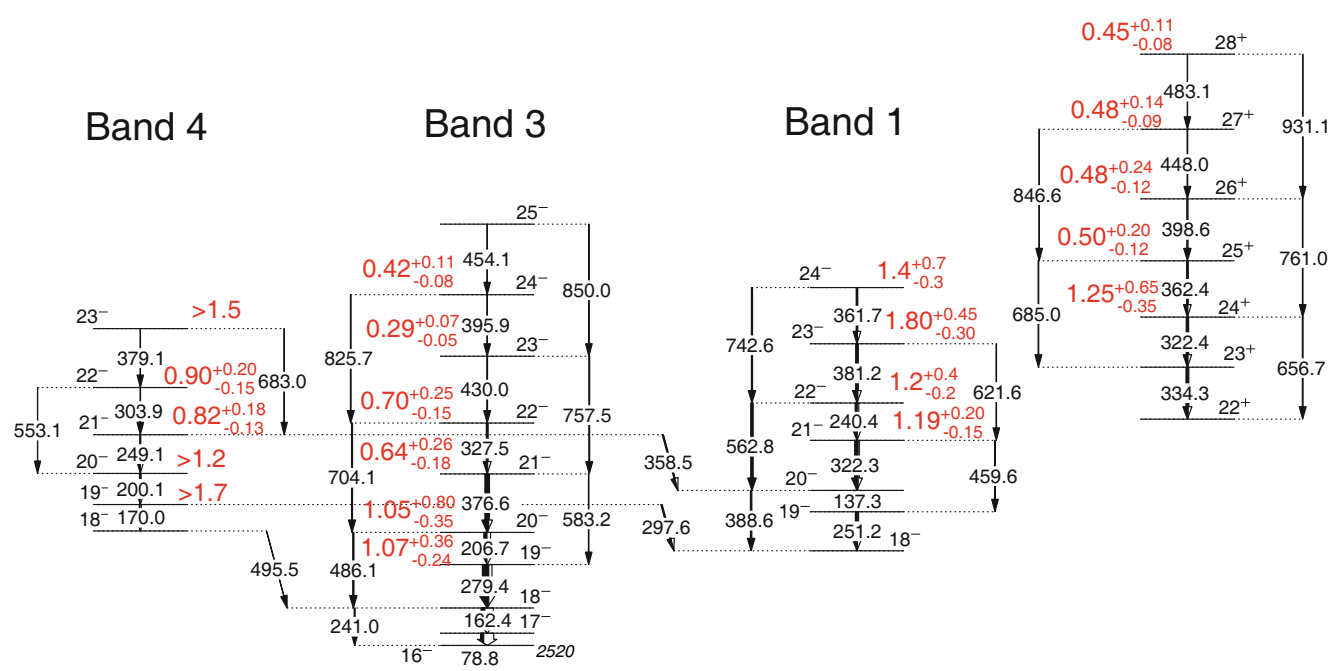

Fig. 3. (Color online) Partial level scheme of the high-energy part of four bands of ${ }^{194} \mathrm{Tl}$, from ref. [5]. The lifetimes (shown in red) are measured in ps.

measurements yield similar results, and an average value of $\tau_{\text {final }}=1.2_{-0.2}^{+0.4} \mathrm{ps}$ for the lifetime of this level is deduced.

Lifetimes were measured for several levels in four rotational bands in ${ }^{194} \mathrm{Tl}$ and results are shown in fig. 3 and in table 1.

The upper part $(I \gtrsim 18)$ of Bands 1 and 4 were assigned a four-quasiparticle $\pi h_{9 / 2} \otimes \nu i_{13 / 2}^{-3}$ configuration, which includes valence nucleons with both particle $\left(\pi h_{9 / 2}\right)$ and hole $\left(\nu i_{13 / 2}^{-3}\right)$ nature. Furthermore triaxial shape was predicted for this configuration in ${ }^{194} \mathrm{Tl}$ [6]. Such features are characteristic for chiral symmetry systems. Most importantly Bands 1 and 4 show excellent near-degeneracy in their excitation energy, alignments and $B(M 1) / B(E 2)$ transition probability ratios [6]. $B(M 1)$ and $B(E 2)$ transition probabilities measurements were carried out in this work and are shown in fig. 4(a) and (b). 
Table 1. $B(M 1)$ and $B(E 2)$ values in W.u. for the transitions in ${ }^{194} \mathrm{Tl}$, deduced from the measured lifetimes shown in fig. 3 . The $\gamma$-ray intensities and multipolarities of the transitions are also shown

\begin{tabular}{|c|c|c|c|c|}
\hline$I_{i}^{\pi}$ & $E_{\gamma}(\mathrm{keV})$ & $I_{\gamma}$ & $\sigma \lambda$ & $B(M 1), B(E 2)$ (W.u.) \\
\hline \multicolumn{5}{|c|}{ Band 1} \\
\hline \multirow[t]{2}{*}{$24^{-}$} & 361.7 & $45(16)$ & M1 & $0.15_{-0.05}^{+0.10}$ \\
\hline & 742.6 & $41(18)$ & $\mathrm{E} 2$ & $11_{-5}^{+8}$ \\
\hline \multirow[t]{2}{*}{$23^{-}$} & 381.2 & $79(28)$ & M1 & $0.19_{-0.04}^{+0.07}$ \\
\hline & 621.6 & $14(3)$ & $\mathrm{E} 2$ & $7.1_{-2.1}^{+5.2}$ \\
\hline \multirow[t]{2}{*}{$22^{-}$} & 240.4 & $78(12)$ & M1 & $0.54_{-0.12}^{+0.22}$ \\
\hline & 562.8 & $89(13)$ & $\mathrm{E} 2$ & $59_{-13}^{+23}$ \\
\hline \multirow[t]{2}{*}{$21^{-}$} & 322.3 & $128(19)$ & M1 & $0.53_{-0.12}^{+0.18}$ \\
\hline & 459.6 & $13(4)$ & $\mathrm{E} 2$ & $32_{-11}^{+18}$ \\
\hline \multicolumn{5}{|c|}{ Band 2} \\
\hline \multirow[t]{2}{*}{$28^{+}$} & 483.1 & $10(5)$ & M1 & $0.24_{-0.09}^{+0.14}$ \\
\hline & 931.1 & $12(5)$ & $\mathrm{E} 2$ & $17_{-6}^{+9}$ \\
\hline \multirow[t]{2}{*}{$27^{+}$} & 448.0 & $18(5)$ & M1 & $0.30_{-0.09}^{+0.16}$ \\
\hline & 846.6 & $16(8)$ & $\mathrm{E} 2$ & $22_{-8}^{+12}$ \\
\hline \multirow[t]{2}{*}{$26^{+}$} & 398.6 & $36(5)$ & M1 & $0.46_{-0.12}^{+0.27}$ \\
\hline & 761.0 & $18(5)$ & E2 & $21_{-6}^{+14}$ \\
\hline \multirow[t]{2}{*}{$25^{+}$} & 362.4 & $46(11)$ & M1 & $0.62_{-0.15}^{+0.40}$ \\
\hline & 685.0 & $22(8)$ & $\mathrm{E} 2$ & $35_{-12}^{+27}$ \\
\hline \multirow[t]{2}{*}{$24^{+}$} & 322.4 & $65(13)$ & M1 & $0.29_{-0.07}^{+0.16}$ \\
\hline & 656.7 & $26(4)$ & $\mathrm{E} 2$ & $13_{-4}^{+8}$ \\
\hline \multicolumn{5}{|c|}{ Band 3} \\
\hline \multirow[t]{2}{*}{$24^{-}$} & 395.9 & $13(2)$ & M1 & $0.33_{-0.08}^{+0.18}$ \\
\hline & 825.7 & $25(8)$ & E2 & $40_{-10}^{+16}$ \\
\hline \multirow[t]{2}{*}{$23^{-}$} & 430.0 & $22(3)$ & M1 & $0.48_{-0.08}^{+0.18}$ \\
\hline & 757.5 & $30(4)$ & $\mathrm{E} 2$ & $81_{-17}^{+28}$ \\
\hline \multirow[t]{2}{*}{$22^{-}$} & 327.5 & $50(6)$ & M1 & $0.53_{-0.12}^{+0.25}$ \\
\hline & 704.1 & $35(7)$ & $\mathrm{E} 2$ & $28_{-7}^{+14}$ \\
\hline \multirow[t]{2}{*}{$21^{-}$} & 376.6 & $138(17)$ & M1 & $0.56_{-0.13}^{+0.32}$ \\
\hline & 583.2 & $16(6)$ & $\mathrm{E} 2$ & $19_{-7}^{+16}$ \\
\hline \multirow[t]{2}{*}{$20^{-}$} & 206.1 & $116(21)$ & M1 & $0.81_{-0.24}^{+0.73}$ \\
\hline & 486.1 & $44(9)$ & E2 & $38_{-13}^{+37}$ \\
\hline \multirow[t]{2}{*}{$19^{-}$} & 279.4 & $236(29)$ & M1 & $0.74_{-0.16}^{+0.34}$ \\
\hline & $(442)$ & $<17$ & $\mathrm{E} 2$ & $<30$ \\
\hline \multicolumn{5}{|c|}{ Band 4} \\
\hline \multirow[t]{2}{*}{$23^{-}$} & 379.1 & $1.6(5)$ & M1 & $<0.15$ \\
\hline & 683.0 & $0.7(4)$ & $\mathrm{E} 2$ & $<10$ \\
\hline \multirow[t]{2}{*}{$22^{-}$} & 303.9 & $1.8(7)$ & M1 & $0.69_{-0.16}^{+0.24}$ \\
\hline & 553.1 & $0.4(1)$ & $\mathrm{E} 2$ & $30_{-9}^{+22}$ \\
\hline $21^{-}$ & 249.1 & $2.4(3)$ & M1 & $0.73_{-0.17}^{+0.30}$ \\
\hline $20^{-}$ & 200.1 & $2.9(5)$ & M1 & $<0.9$ \\
\hline $19^{-}$ & 170.0 & $2.1(4)$ & M1 & $<0.7$ \\
\hline
\end{tabular}

All measured values for the $B(M 1)$ and the $B(E 2)$ transition probabilities in Bands 1 and 4 look very similar (see fig. 4(a) and (b)). This strongly supports the suggested chiral symmetry of the two bands, and highlights the excellent near-degeneracy of this pair, making it perhaps the best chiral symmetry pair known to date.

The measured transition probabilities helped to reveal more details on the nature of the third negative-parity band, Band 3. This band was also assigned a $\pi h_{9 / 2} \otimes \nu i_{13 / 2}^{-3}$ configuration [5], and it shows very similar $B(M 1)$ and $B(E 2)$ transition rates to those of Bands 1 and 4 . The observed similarity in the $B(E 2)$ rates indicates no change in the nuclear shape and is in accordance with Band 3 corresponding to a triaxial shape and being a part of a second chiral system in this nucleus. To determine the expected features of multiple bands built on the $\pi h_{9 / 2} \otimes$ $\nu i_{13 / 2}^{-3}$ configuration, Many Particle Rotor (MPR) model calculations [19] were performed.

The MPR model assumes a Nilsson potential with standard parameters [20] for the potential of the valence nucleons. The $\pi h_{9 / 2} \otimes \nu i_{13 / 2}^{-3}$ configuration of the negativeparity bands was described in the MPR calculations as one proton placed among the 10 orbitals of the $\pi h_{9 / 2}$ shell and 11 neutrons placed among the 14 orbitals of the $i_{13 / 2}$ shell. The g-factor of the core was taken as $g_{R}=0.3$, and the $\mathrm{g}$-factors of the odd proton and neutrons were calculated assuming an attenuation of the g-factors of free nucleons, $g_{s}=0.7 g_{\text {free }}$. Taking into account the Cranked Nilsson Strutinsky calculations for this configuration [6] a quadrupole deformation of $\varepsilon=0.15$ and a triaxial deformation of $\gamma=40^{\circ}$ were employed. An irrotational moment of inertia was used. The results are shown in fig. 4 .

Figure 4(d) shows the calculated excitation energies of the four lowest-energy bands which have similar features to the experimental energies, fig. 4(c). Furthermore an excellent agreement was found between the measured and the calculated values of the $B(M 1)$ and $B(E 2)$ reduced transition probabilities, see fig. 4(a) and (b), particularly for $I<23$, where the interaction of these same-parity bands is not expected to play a significant role.

The theoretical results were examined to determine whether all calculated bands correspond to chiral geometry. It was found that the expectation values of the angles between the proton, neutron and collective rotation angular momenta are large and indicate a three-dimensional geometry. Furthermore the total angular momenta projections along the three major nuclear axes remain large for all calculated bands. It was concluded that the calculated bands correspond to three-dimensional chiral geometry, thus supporting the proposed chiral symmetry for the negative parity bands in ${ }^{194} \mathrm{Tl}$.

Should Band 3 have a chiral partner? A multiplet of two chiral systems, built on the same configuration of two valence nucleons, behaving as a particle and a hole in the same single shell, generates four bands arranged in two distinct pairs $[1,21]$. However, when the configuration is allowed to span a larger configuration space than a single orbital per nucleon, the partner bands in the pair show distinct differences $[22,23]$, including increased relative 

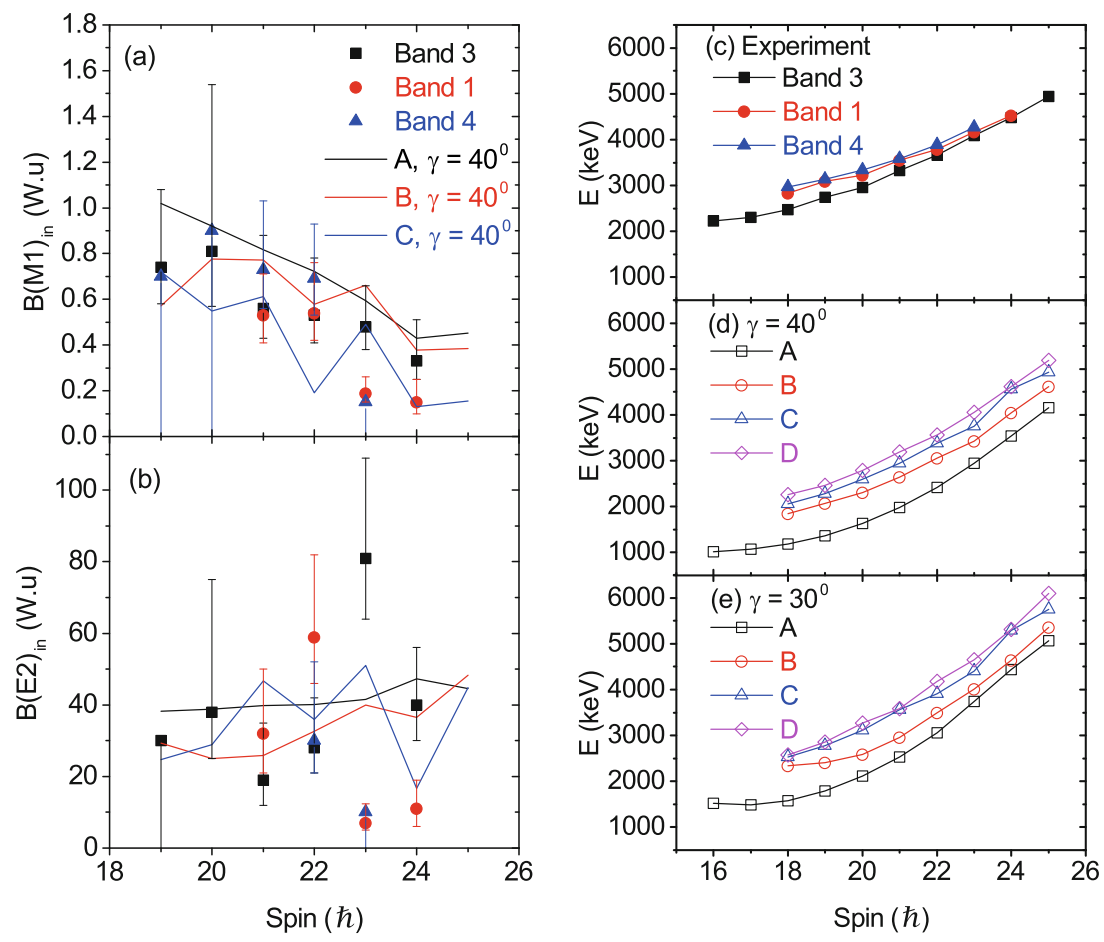

Fig. 4. (Color online) Experimental and calculated (a) $B(M 1)$, and (b) $B(E 2)$, transition probabilities in the negative-parity bands of ${ }^{194} \mathrm{Tl}$. Measured excitation energies are shown in (c). The calculated energies for the four lowest-energy bands and for $\gamma=40^{\circ}$ and $\gamma=30^{\circ}$ are plotted in (d) and (e), respectively. The calculated bands are labeled with A, B, C and D according to their excitation energy.

excitation energies. Consider for instance the four calculated bands in ${ }^{194} \mathrm{Tl}$ for the largest triaxiality $\gamma=30^{\circ}$, fig. 4(e). Note that the yrast chiral pair shows large relative excitation energy between the partners, while the yrare chiral pair does not. At $\gamma=40^{\circ}$ the partner band from the yrast chiral pair reaches an excitation energy similar to that of the yrare chiral pair. This means that assuming that the calculations describe well the nature of the experimental bands, the partner of Band 3 is expected at excitation energy similar to that of the yrare chiral pair, i.e. near Bands 1 and 4. These two bands are already nonyrast and rather weakly populated, thus should a fourth band exist at similar excitation energy it may remain unobserved in our data.

In summary, lifetime measurements were performed for four bands in ${ }^{194} \mathrm{Tl}$, including the four-quasiparticle chiral pair with close near-degeneracy. The extracted reduced $B(M 1)$ and $B(E 2)$ transition probabilities in this pair confirm the excellent near-degeneracy of these bands and suggest that this chiral pair is perhaps the best chiral system found to date. The third negative-parity band, Band 3, may also correspond to chiral geometry, and could be a part of a chiral multiplet built on the $\pi h_{9 / 2} \otimes \nu i_{13 / 2}^{-3}$ configuration.

We would like to thank the crew of the iThemba LABS separated sector cyclotron for the delivery of the oxygen beam. Our sincere gratitude goes also to Ingemar Ragnarsson and Gillis Carlsson, for sharing the codes of the Cranked Nilsson Struti- nsky and the Multiparticle Rotor Models, and for numerous discussions. This work is based upon research supported by the National Research Foundation, South Africa, with grants UID 76632 and 93531, and by an International Science and Technology Agreement of South Africa and Russia with grants UID 75248 and RFBR11-02-93963-SA_a.

Open Access This is an open access article distributed under the terms of the Creative Commons Attribution License (http://creativecommons.org/licenses/by/4.0), which permits unrestricted use, distribution, and reproduction in any medium, provided the original work is properly cited.

\section{References}

1. S. Frauendorf, J. Meng, Nucl. Phys. A 617, 131 (1997).

2. D. Tonev et al., Phys. Rev. Lett. 96, 052501 (2006).

3. A.D. Ayangeakaa et al., Phys. Rev. Lett. 110, 172504 (2013).

4. I. Kuti et al., Phys. Rev. Lett. 113, 032501 (2014).

5. P.L. Masiteng et al., Eur. Phys. J. A 50, 119 (2014).

6. P.L. Masiteng et al., Phys. Lett. B 719, 83 (2013).

7. R.T. Newman et al., Balkan Phys. Lett. Special Issue, $182(1998)$

8. J.F. Sharpey-Schafer, Nucl. Phys. News 14, 5 (2004).

9. I.Kh. Lemberg, A.A. Pasternak, Modern Methods of $\mathrm{Nu}$ clear Spectroscopy (Nauka, Leningrad, 1985).

10. E. Grodner et al., Eur. Phys. J. A 27, 325 (2006).

11. J. Srebrny et al., Nucl. Phys. A 683, 21 (2001). 
12. R.M. Lieder et al., Eur. Phys. J. A 21, 37 (2004).

13. E.O. Lieder et al., Eur. Phys. J. A 35, 135 (2008).

14. E.O. Lieder et al., Nucl. Instrum. Methods A 607, 591 (2009).

15. E.O. Lieder et al., Phys. Rev. Lett. 112, 202502 (2014).

16. E. Grodner, I. Sankowska, T. Morek, S.G. Rohoziński, Ch. Droste, J. Srebrny, A.A. Pasternak, M. Kisieliński, M. Kwalczyk, J. Kownacki, J. Mierzejewski, A. Król, K. Wrzosek, Phys. Lett. B 703, 46 (2011).
17. C. Mihai et al., Phys. Rev. C 81, 034314 (2010)

18. I. Sankowska et al., Eur. Phys. J. A 37, 169 (2008).

19. B.G. Carlsson, I. Ragnarsson, Phys. Rev. C 74, 044310 (2006).

20. T. Bengtsson, I. Ragnarsson, Nucl. Phys. A 436, 14 (1985).

21. Ikuko Hamamoto, Phys. Rev. C 88, 024327 (2013).

22. E.A. Lawrie, O. Shirinda, Phys. Lett. B 689, 66 (2010).

23. O. Shirinda, E.A. Lawrie, Eur. Phys. J. A 48, 118 (2012). 